\title{
Efficient Joining and Leaving for Receiver Driven Multicast Congestion Control
}

\author{
Karan Singh \\ School of Information and Communication Technology \\ Gautam Buddha Univesity, Greater Noida \\ UP, India, - 201310
}

\author{
Rama Shankar Yadav \\ Department of Computer Science and Engineering \\ Motilal Nehru National Institute of Technology, Allahabad, \\ UP, India, - 21004
}

\begin{abstract}
Multimedia applications such as video conferencing, multiparty video games, military applications news feeds, video-audio transmission and IP TV etc. are today and next generation demand in our life. Multicast communication is better than broadcast and unicast as communication scheme to handle above stated application. Multicast is one to group communication whereas every one knows the multicast group address and uses the UDP protocol. So, it suffers with flooding or congestion problem. Many research groups have proposed the mechanism to control the congestion in multicast. The congestion control schemes are based on source driven, receiver driven and hybrid. Our proposed work is receiver driven approach and we are providing efficient joining and leaving scheme for multicast congestion control which is based on adaptive throughput. In this scheme, we are going to proposed multiple layered joining and leaving approach whereas leaving decision is based on adaptive deaf concept. We have analysis the simulation results using NS-2 which show that performance and packet loss of purposed approach are better than existing approach.
\end{abstract}

\section{Categories and Subject Descriptors}

C.2.3 [Communication and Network]: Multicast Congestion Control

\section{General Terms}

Measurement and Performance.

\section{Keywords}

Congestion, IP multicast, IGMP, Multicast, Multicast Routing

\section{INTRODUCTION}

Network is very interesting area in research field which are wired and wireless, both networks provide the data transmission via unicast, broadcast and multicast. In case of unicast one source send a data copy to only one destination while in broadcast one sender sends the data copy to all connected receiver at a time. Multicast is special class of broadcast where one or many source send a data stream to a particular group of receivers. Multicast is efficient then unicast and broadcast whereas it is demand of multimedia applications. But it suffers with various problems such as congestion, security, reliability etc. where congestion have important role to increase or decrease the performance of multicast communication. Congestion [1][2] in network occurs when increment in network load either leads only to small increases in network throughput, or reduction in network throughput. The main reasons behind congestion are memory space, buffer, channel bandwidth, processor capacity, number of users (network load), link failure etc.
There are many approaches which manage the congestion in multicast communication known by source driven [12] and receiver driven [7]. In source driven, a source takes active role to control the congestion but it has problem of feedback suppression and underutilizing of receivers. In case of receiver driven approach, receivers take decision according to their own capacity. This approach is source dived the stream into various sub stream called layers [3] (base layer and enhance layer) whereas receiver joins or leave the layer based on available capacity. The authors provide the cumulative, in which receiver join layer in increment order (base layer then enhance layers) whereas leave the layers in reverse order and in case of non-commutative receiver joins or leave the layers without order. There are may approach QIRLM [14], PLM [9], TFMCC [8], MILDA [13], RLM [10], RLC [4], FLID-DL[6], SMCC [5], ESMCC [15] etc. which are using single layer joining approach at a time and direct leave the layer which are reason for underutilization of resources and provide the less performance. In this paper we are going to propose a efficient joining and leaving approach to adaptive throughput which increase system performance and control the congestion efficiently.

The rest of the paper is organized as follows. Section 2 deals with related work whereas section 3 details the proposed solution and section 4 discussion with analysis of results. Finally, section 5 deals with concluding remarks

\section{RELATED WORK}

Our approach is based on receiver driven layered approach. There are many schemes available in this category such as RLM [10], RLC [4], FLID-DL [6], TFMCC [8], SMCC [5], ESMCC [15] etc. The brief description of related propose approach are following

Receiver driven Multicast layered (RLM) [10] is a transmission scheme which use layered approach for stream transmission. In this transmission scheme flow of information is divided in several layers. Each layer is to be transmitted in separate multicast group to a set of receiver. Base layer insure the initial quality of reception. Each addition layer join will increase the reception quality of group of receiver. RLM adapt an approach to carry out active experiment by addition a layer at fixed time. This addition of layer is called join experiment. If join experiment cause congestion (single packet loss) in network receiver drop the recently added layer and if join experiment become successful it wait for a fixed amount of time and do join experiment again for joining next layer. RLM scheme is scalable as well as able to handle the heterogeneous receiver. This scheme suffers with problem of co-ordination between the receivers. Although share learning process is used by RLM can co-coordinate receiver locally. This scheme dose not takes any incentive to control congestion during IGMP Leave Latency period. 
Receiver driven layered congestion Control (RLC) [6] tried to sort out the problem of RLM so that it is called as successor of receiver driven layered multicast. As original RLM scheme suffer with the problem of co-ordination as well as IGMP leave latency problem. Basic Concept (join experiment) remains same in this scheme as compare to RLM. However the approach includes three major changes regarding the detection of spare bandwidth, the synchronization of experiment and the avoidance of leave latency. First change is detecting available bandwidth in the network, sender initiated probes are introduce which are sent out by the sender. Second change introduces synchronization point by tagged data packet to synchronize join experiment. This improves the RLM since all the downstream receivers are synchronized instead of local once only. Third change deal with avoidance of the leave latency effect, caused by the large response time of a leave operation. For that a deaf period is define during which a receiver dose not react to further losses for a defined time. Subscriptions levels can increase only at synchronize points, and decrease at any time. Decrease if a loss is experienced during normal transmissions. Increase at a SP if no losses are experienced during the burst preceding that SP. Unchanged otherwise.

Fair Layer Increase Decrease- Dynamic layer (FLID-DL) [6] is a protocol for improving RLC, which is introduces the concept of Dynamic Layering (DL) to reduce the IGMP leave latencies. With such a dynamic layering, a receiver can reduce its reception rate simply by not joining any additional layer. FLID-DL has a few improvements over RLC, but it still exhibits not very good fair behavior towards TCP sessions.

TCP-friendly multicast congestion control (TFMCC) [8]. With single rate congestion control schemes, the sender transmits at a rate requested by the slowest receiver in the group. While these protocols are not designed to scale to large sets of receivers with heterogeneous available bandwidth there is building consensus that these protocols are sufficiently mature and well tested for Internet deployment.

Smooth Multirate Multicast Congestion Control (SMMCC) [5] is extension of TFMCC friendliness and smooth rate control due to long term averaging of loss and RTT measurements. Each layer of SMCC transmits at a rate within a designated interval according to participants of that layer. If the expected throughput computed is above the maximum sending rate of its current subscription level, the receiver initiates a joint attempt. During the joint attempt, instead of join the next layer, the receiver increase slowly it reception rate (by one packet per RTT). When it reaches the target rate of the next layer, it then joins this layer and drops the special additive increase layers. The join attempt is ceased if a loss event occurs meanwhile.

Extended Smooth Multirate Multicast Congestion Control (ESMCC) [15] is extension of SMCC which tackle the problem of SMCC using adaptive RTT. In this approach joining or leaving operation are based on RTT. These multicast congestion control approach are receiver driven and joining single layer while leave the layer due to overloading. So, these are suffered with less performance of resource. In next section, we are going to propose an efficient layered joining and leaving according to adaptive throughput

\section{PROPOSED WORK}

In this section we describe the architecture, motivation and proposed algorithm known by efficient joining and leaving for multicast congestion control (EJLMCC) which is following

\subsection{Architecture}

We are using the architecture for our approach which is shown in the figure 1. It consists of one sender who sends the packets stream through IP Layered-multicast to the receivers. IP Multicast [20] is an advanced group communication mechanism designed to operate on the Internet Protocol (IP) [18] where each incoming layer to multicast router form group. When a packet is sent to the group, all members of the group receive the packet. If there are $N$ $>1$ recipients behind a link, then unicast communication would require $\mathrm{N}$ packets with the same content to be sent explicitly to each of the $\mathrm{N}$ recipients. Multicast requires the packet to be forwarded only once. In our architecture, receiver receives packet stream by joining multicast group. In architecture $R_{1}, R_{2} . . R_{k}$ consist for $\mathrm{k}$ number of receiver. As this approach is scalable for receiver, $\mathrm{k}$ could be any number between 1 and 100 .

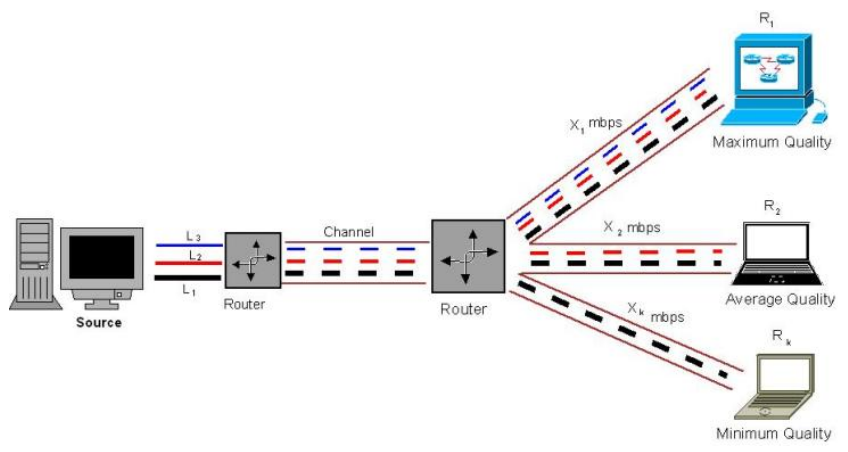

Figure 1: Architecture

If the receivers have the capability to receive all the layer of data packets, then the quality of received stream will be high. Every receiver, sender as well as router have buffer to schedule incoming packet. If receiver does not have capability to accept all streams sent by source, then quality of received stream will be degraded. $L_{1}, L_{2}, L_{3}$ show the encoded layer and $X_{1}, X_{2} \ldots X_{k}$ showing the link bandwidth of each receiver associated with router Every Receiver join layer by doing join experiment depends on the capacity of receiver. If there is no congestion during join experiment receiver will join layer otherwise leave highest layer Figure 2 show that every multicast router maintains a multicast group. It use most general protocol for multicast routing [18] called Distance vector multicast routing protocol (DVMRP) [19] used. DVMRP use "broadcast \& prune" approach for multicast routing. It builds per-source broadcast trees based upon routing exchanges, then dynamically creates per-sourcegroup multicast delivery trees by pruning (removing branches from) the source's truncated broadcast tree. It performs reverse path forwarding (RPF) to determine when multicast traffic should be forwarded to downstream interfaces. In this way, source-rooted shortest path trees can be formed to reach all group members from each source network of multicast traffic. IGMP[17] protocol is used to manage the multicast group. Receiver use joins and leave message to join are leaving a particular group. 


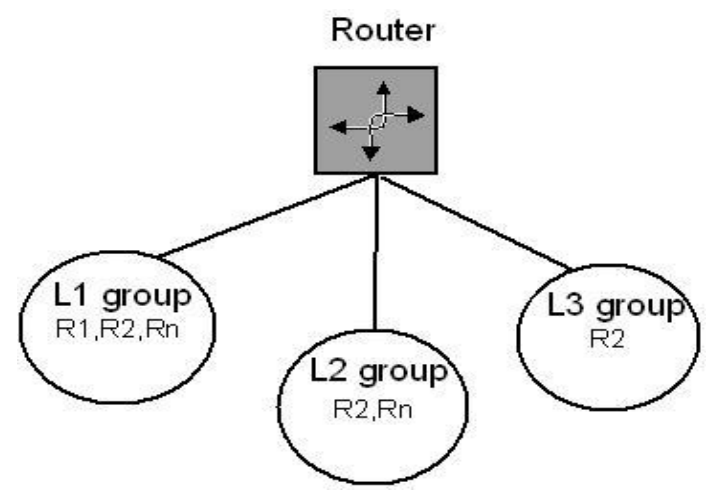

Figure 2: Group Management

Group maintenance is cumulative and dynamic in nature. Every receiver will join base layer $\mathrm{L}_{1}$ at the starting then move next layer depend upon the capacity of bandwidth and congestion state. There may be many receivers possible in our architecture. The entire receiver first join base layer that provide initial quality. To enhance quality of stream receiver further go for join next immediate layer.

\subsection{Motivation}

We have proposed new scheme to perform layer joining and leaving operation whereas receiver joins multiple layer instead of single layer at a time and leave a layer according to adaptive deaf concept. We have compare the performance to existing approach such RLM [10] SMCC [5], ESMCC [15] but we are taking receiver driven layered multicast (RLM) as an example to describe our approach. RLM is adaptive approach to carry out active experiment by addition a layer at fixed time. This addition of layer is called join experiment. If join experiment cause congestion in network receiver drop the recently added layer and if join experiment become successful it wait for a fixed amount of time and do join experiment again for joining next layer. For example in figure 3, stream is divided into 4 layers named as $L_{1}, L_{2}, L_{3}, L_{4}$ and initially receiver receives the base layer $\left(L_{1}\right)$ while a layer $L_{i}$ has data rate $R_{i}$. At time $t_{1}$, receivers do experiment for checking the packet loss and it experience that there is no packet loss. So, receiver joins the layer $L_{2}$ and wait a period $(\mathrm{P}=\mathrm{T})$ for next experiment.

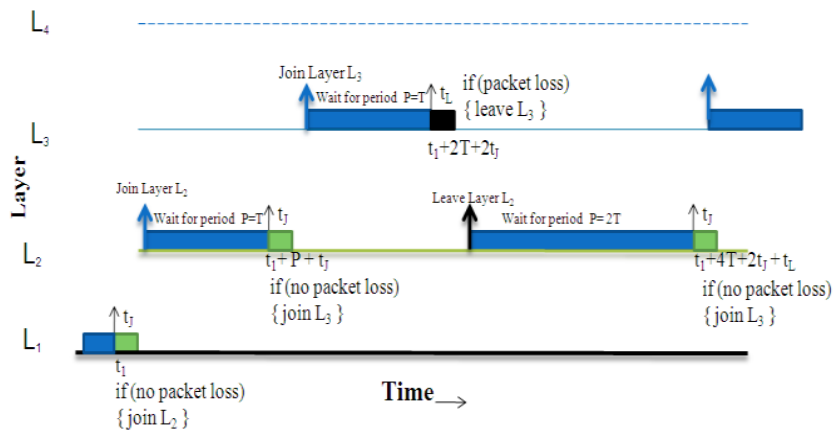

Figure 3: Existing Approach

Similarly, at time $\mathrm{t}=t_{1}+P+t_{j}$ (where is joining time) receivers join the layer $L_{3}$ but at time $t=t_{1}+2 T+2 t_{j}$ receiver suffers from packet loss than it leave the layer and increase the waiting period $(\mathrm{P})$ by two times. Now receiver experiment at time $t=t_{1}+4 T+2 t_{j}$ for packet loss and it feel that there is no packet loss so it again joins the $L_{3}$. Similar trend follow for all join and leaving operation. Suppose received throughput 3.2 mbps, layers $L_{1}, L_{2}, L_{3}, L_{4}$ rate are $0.5,1.0,1.5,2.0$ and period of decision (T) is $10 \mathrm{~ms}$. We can observed that at time $t_{1}=0.1$ and $t=t_{1}+2 T+2 t_{j}=20.1$ (if joining time is negligible), there is no packet loss during this period and average throughput is $(1.5 * 10+3.0 * 10) \mathrm{mbps} / 20 \mathrm{~ms}=2250 \mathrm{mbps}$. If we join the multiple layers then average throughput will be $3000 \mathrm{mbps}$. If receivers leave the layer at same time then average throughput will decease more. Thus we can achieve better throughput using multiple layer joining and adaptive decision for leave layers. In the next paragraph, we are going to details the proposed multiple layer joining approach

\subsection{Contributed Work}

The The proposed approach is based on judicially decision of adaptive throughput whereas a receiver calculated throughput $\left(\mathrm{Th}_{\text {Cal }}\right)$ and received throughput $\left(\mathrm{Th}_{\mathrm{Recv}}\right)$ according to equation 1 $[21,22,23]$ and 2 with respectively whereas receiver take decision each experiment time known by period $\mathrm{P}(\mathrm{T})$.

$$
T h_{C a l} \approx \frac{P_{S}}{R T T * \sqrt{P L R}}
$$

$$
T h_{\operatorname{Re} c v}=\sum_{1=L}^{i=1} R_{i}
$$

Where RTT $=$ Rount Trip Time

PLR, = Packet Loss Ratio

$\mathrm{P}_{\mathrm{S}}=$ Packet Size

$\mathrm{R}_{\mathrm{i}}=$ Rate of $\mathrm{i}^{\text {th }}$ layer

Thus, receiver calculates the RTT and PLR at each period to find out the calculated throughput and it measure the received throughput using joined layers. The leaving and joining operation are based on adaptive decision these two throughput which is discussed in next section.

\subsubsection{Layer Joining Scheme}

We are proposing new layer joining scheme which multiple layer joining. Receiver takes the adaptation using calculated throughput $\left(\mathrm{Th}_{\mathrm{Cal}}\right)$ and received throughput $\left(\mathrm{Th}_{\mathrm{Recv}}\right)$. In others hand it join the multiple layers continuous according to equation 3 .

$$
T h_{\text {Cal }} \geq T h_{\text {Recv }} \text { i.e. Layer Join Decision }
$$

If equation 3 is satisfied, receivers joint the multiple layers whereas it is not satisfied (congestion may be occurred using buffer overflow) than stop to joining layers and use leave decision (section 3.3.2). The proposed approach can be illustrates by figure 4. Suppose received throughput is $3.2 \mathrm{mbps}$ (using equation 1 at RTT and PLR value), layers $L_{1}, L_{2}, L_{3}, L_{4}$ rate are $0.5,1.0,1.5$, 2.0 and period of decision (T) is $10 \mathrm{~ms}$. At time $t_{1}=0.1 \mathrm{~ms}$, check the calculated i.e. 3.2 while receiver joins the layer $L_{1}, L_{2}, L_{3}$ because received throughput of layers combination $L_{1}(0.5 \mathrm{mbps})$, $L_{1}, L_{2} \quad(1.5=0.5+1.0), L_{1}, \quad L_{2}, L_{3}(3.0=0.5+1.0+1.5) \quad$ and $L_{1}, L_{2}, L_{3}, L_{4}(5.0=0.5+1.0+1.5+2)$. So, receiver stops 
joining operation at layer $L_{3}$. Now receivers receive the throughput using $L_{1}, L_{2}, L_{3}$ till period of decision finished and check the buffer status for congestion (packet loss).

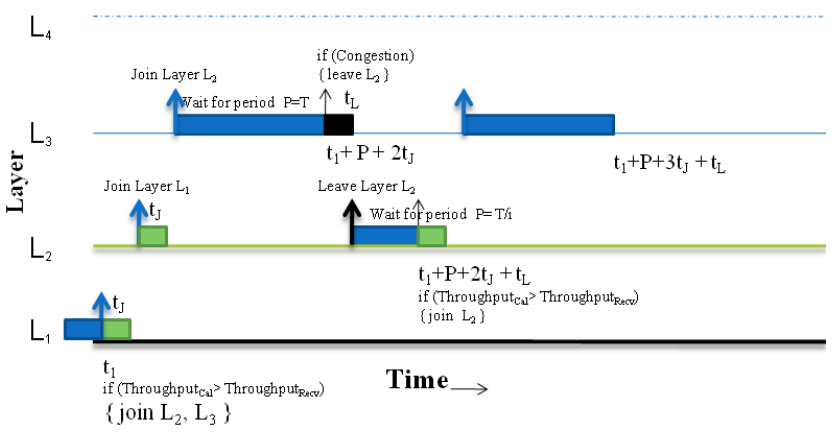

Figure 4: Layer Joining Approach

At time $t=t_{1}+2 T+2 t_{j}$ receiver feel that packet are lost. So it perform the leave operation such as leaves the layer $\left(L_{3}\right)$. From table 1 , we can observed that proposed method throughout at $\mathrm{t}=$ 30 is $134 \mathrm{mbps}$ and $33.90 \mathrm{mbps}$ in existing i.e. proposed approach is better than existing approach. In our approach probability of packet loss increase but it can be handling with adaptive leaving concept for leave layers. In next section we will discuss the layer leaving scheme.

\subsubsection{Layer Leaving Scheme}

Proposed leaving layer scheme is also based on adaptive throughput with additional feature. Receiver take join or leave decision according to equation 3 . If it is not satisfied then receiver checks the status of congestion due join congestion (due to overflow). Now if receiver feels the congestion it takes others adaptive decision leaves layer or deaf period concept whereas adaptive decision is taking between overloaded throughput $\left(\mathrm{Th}_{\text {Over }}\right)$ and threshold value of overloaded throughput $\left(\mathrm{Th}_{\mathrm{T}}\right)$. The overload throughput is difference between calculated throughput and received throughput can be seen equation 4 .

$$
\mathrm{Th}_{\text {Over }}=\mathrm{Th}_{\text {Recv }}-\mathrm{Th}_{\mathrm{Cal}}
$$

where as threshold value $\left(\mathrm{Th}_{\mathrm{T}}\right)$ is normalizing form of highest layer which is calculated $\left(\mathrm{Th}_{\mathrm{T}}=L_{R H} / \rho\right)$ using divide the higher layer rate $\left(L_{R H}\right)$ by normalize factor $(\rho)$ and $\rho$ is constant value such $1,2.3,4 \ldots . . n$. The values of $\rho$ is based on history if there are some burst loss in network then we take small value of $\rho$ ( 1 or 2 ), otherwise we take big value of $\rho$ ( 8 or 9$)$. In proposed approach, receiver takes the decision for deaf or leave layer according to equation 5 .

$$
\mathrm{Th}_{\text {Over }} \leq \mathrm{Th}_{\mathrm{T}} \text { i.e. deaf for layer }
$$

If receiver satisfy the equation 5 then it go in deaf state (receiver will not receive any packets) for deaf period whereas deaf period $\left(D_{f}\right)$ is ratio of experiment time $\mathrm{P}(\mathrm{T})$ and number of layer (i) i.e. $\frac{T}{i}$. If receiver will not satisfy the equation 4 then leaves the layer and decrease the rate of that layer by $\rho$ during leave period $\left(t_{L}\right)$ while update the experiment time (P) by T-D (if receiver deaf in pervious experiment). The proposed approach can be illustrates by figure 5. Suppose calculated throughput is $3.2 \mathrm{mbps}$, layers $L_{1}, L_{2}, L_{3}, L_{4}$ rate are $0.5,1.0,1.5,2.0$ and period of decision (T), join latency and leave latency are $10 \mathrm{~ms}, 0.2 \mathrm{~ms}, 1.0 \mathrm{~ms}$ respectively. At time $t_{1}=0.1$, receiver joins the layer $L_{1}, L_{2}, L_{3}$.

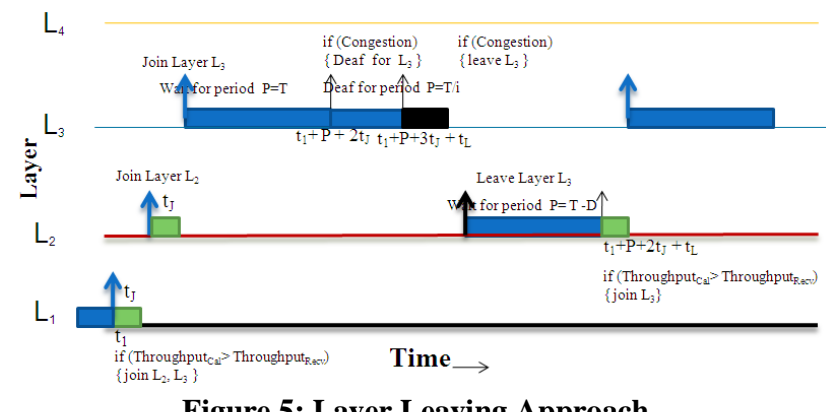

Figure 5: Layer Leaving Approach

Now, at time $t=t_{1}+2 T+2 t_{j}$ receiver checks the buffer status for congestion (packet loss) and feels the congestion. So, $t=t_{1}+$ $2 T+2 t_{j}=15, \mathrm{Th}_{\mathrm{Cal}}$ is updates $2.6 \mathrm{mbps}$ due to congestion. So it checks the overloaded condition $\left(\mathrm{Th}_{\text {Over }}\right.$ and $\mathrm{Th}_{\mathrm{T}}$ are $0.4,0.5$ whereas value of $\rho$ is 3 ) is true. So, receivers go in deaf period. When deaf period finished, At time $t=t_{1}+P+2 t_{j}+D_{f}=$ $18.3\left(15+\frac{10}{3}\right), \mathrm{Th}_{\mathrm{Cal}}$ is updates $2.4 \mathrm{mbps}$ due to decreasing load. So, it checks again overloaded condition $\left(\mathrm{Th}_{\mathrm{Over}}\right.$ and $\mathrm{Th}_{\mathrm{T}}$ are $0.6,0.5)$ is false. So, receivers go to leave the layer and decrease the rate of highest layer by $0.5 \mathrm{mbps}$.

TABLE 1: Comparison of Approach

\begin{tabular}{|c|c|c|c|c|}
\hline & \multicolumn{2}{|c|}{ Exist Approach } & \multicolumn{2}{c|}{ Proposed Approach } \\
\hline $\begin{array}{l}\text { Tim } \\
\text { e (s) }\end{array}$ & $\begin{array}{l}\text { Throughpu } \\
\mathrm{t}(\text { MBPS })\end{array}$ & $\begin{array}{l}\text { Packet } \\
\text { loss }\end{array}$ & $\begin{array}{l}\text { Throughp } \\
\text { ut(MBPS) }\end{array}$ & Packet loss \\
\hline 1 & 0.45 & 0 & 3.00 & 0 \\
\hline 5 & 2.45 & 0 & 23.00 & 0 \\
\hline 10 & 4.50 & 0 & 48.00 & 0 \\
\hline 15 & 11.90 & 0 & 73.00 & 0 \\
\hline 20 & 16.40 & 0 & 98.00 & 8 \\
\hline 25 & 28.90 & 0 & 119.00 & 0 \\
\hline 30 & 33.90 & 0 & 134.00 & 0 \\
\hline 35 & 58.88 & 0 & 159.05 & 0 \\
\hline 40 & 83.88 & 10 & 184.05 & 6 \\
\hline 45 & 104.38 & 0 & 205.05 & 0 \\
\hline
\end{tabular}

Our proposed work is increasing the performance using multiple join multiple layer and avoid the leaving of layer using adaptive concept of deaf. The complete algorithm of proposed work can be seen in Appendix A. In next section we will discuss the results and analysis of proposed work

\section{RESULTS AND ANALYSIS}

We have simulated the results of proposed approach from Network Simulator NS-2 version 2.30[11].

\subsection{Experiment Topology}

Figure 3 is describing a network topology for proposed work. In this topology one source which is node 0 . Node 1, 2 are working as router and node 3, 4, 5 are receiver1.6 s. We have attached three UDP agents which connected to corresponding CBR and 
providing three layers. Receiver 1 join group 0 such as receiver 1 , 2 have joined group 1, 2.

The bandwidth of channel is 1.6 to $2.0 \mathrm{Mb}$ and delays of channel are $10-50 \mathrm{~ms}$. The layers $\left(\mathrm{L}_{1}, \mathrm{~L}_{2}, \mathrm{~L}_{3}, \mathrm{~L}_{4}\right)$ rates are $0.5,1.0,1.5,2.0$ $\mathrm{mb}$ while queue maximum size is $6 \mathrm{MB}$ where $\mathrm{Q}_{\mathrm{TH}}$ is $80 \%$ of queue size

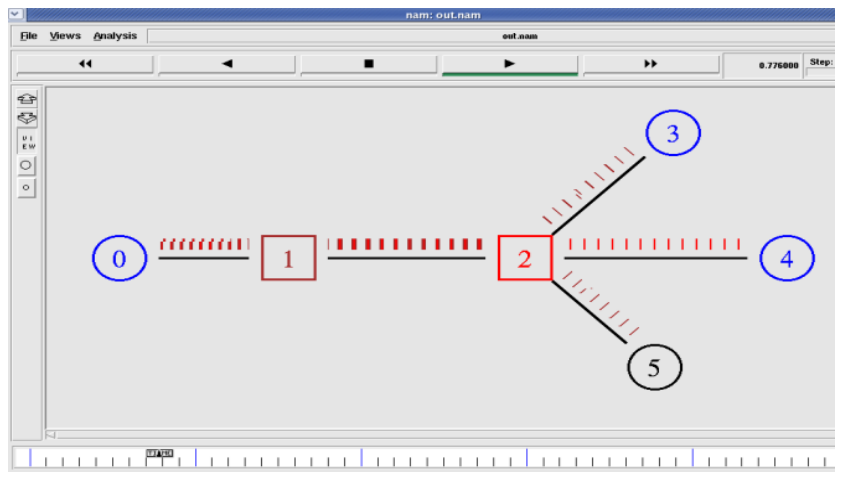

Figure 6: Experiment Topology

\subsection{Experiment Parameter}

We have analyzed packet loss and throughput using various parameters by simulation. In following section, we will discuss throughput and packet loss at different time. Simulation parameters are given in table 2.

TABLE 2: Terminology

\begin{tabular}{|l|l|}
\hline Terms & Significance \\
\hline Packet Size $(\mathrm{Ps})$ & $800-1000$ Byte \\
\hline Queue Size $\left(\mathrm{Q}_{\mathrm{Max}}{ }^{\prime}\right.$ & $4-10(\mathrm{MB})$ \\
\hline Thesold Value of Queue Size $\left(\mathrm{Q}_{\mathrm{TH}}\right)$ & $80 \%$ of $\mathrm{Q}_{\mathrm{Max}}$ \\
\hline Rate of $\mathrm{i}^{\text {th }} \mathrm{R}_{\mathrm{i} \mathrm{i}}$ Layer $^{\text {Layer Join Time }\left(\mathrm{T}_{\mathrm{J}}\right)^{\prime}}$ & $0.5,1.0,1.5,2.0$ \\
\hline Layer & $0.1 \mathrm{~ms}$ \\
\hline Layer Leave Time $\left(\mathrm{T}_{\mathrm{L}}{ }^{\prime}\right.$ & $1.0 \mathrm{~ms}$ \\
\hline Number of Layer $(\mathrm{i})$ & $1,2,3,4$ \\
\hline Initiation Time $\left(\mathrm{T}_{1}\right)$ & $0.1 \mathrm{~ms}$ \\
\hline Network Bandwidth $\left(\mathrm{N}_{\mathrm{B}}\right)$ & $1.6-2.0(\mathrm{MB})$ \\
\hline Network Delay $\left(\mathrm{L}_{\mathrm{D}}\right)$ & $10-50(\mathrm{~ms})$ \\
\hline Simulation Time & $10 \mathrm{~s}$ \\
\hline
\end{tabular}

\subsection{Result Analysis}

In this section we are going to discuss the result analysis. We have analyzed the packet loss and throughput for proposed and existing approach at different parameters. The details decisions are following

In figure 7 graphs plotted between Throughput and number of layer joining which shows receivers status of throughput after joining layers. The graph is showing general behavior of layer joining, if receiver joins more layers than it will receive more throughput. In others hand, if receiver received the less number of layer than it receive less throughput. Here, receiver 1 throughput is less because it have joined one layer and receiver have maximum throughput (5 mbps) because it joined four layers.

In figure 8 graphs plotted between throughput and time which shows current time status of throughput at different period of experiment. At time $20 \mathrm{~s}$ when periods are 5, 10 and 15 seconds then throughput are 1.5, 3.0 and 5 mbps respectively. The graph show that if we increase the period of experiment than performance will be decrease because joining and leaving will be late. So, receivers will receive data from one group of layer more time or loss due to congestion more time.
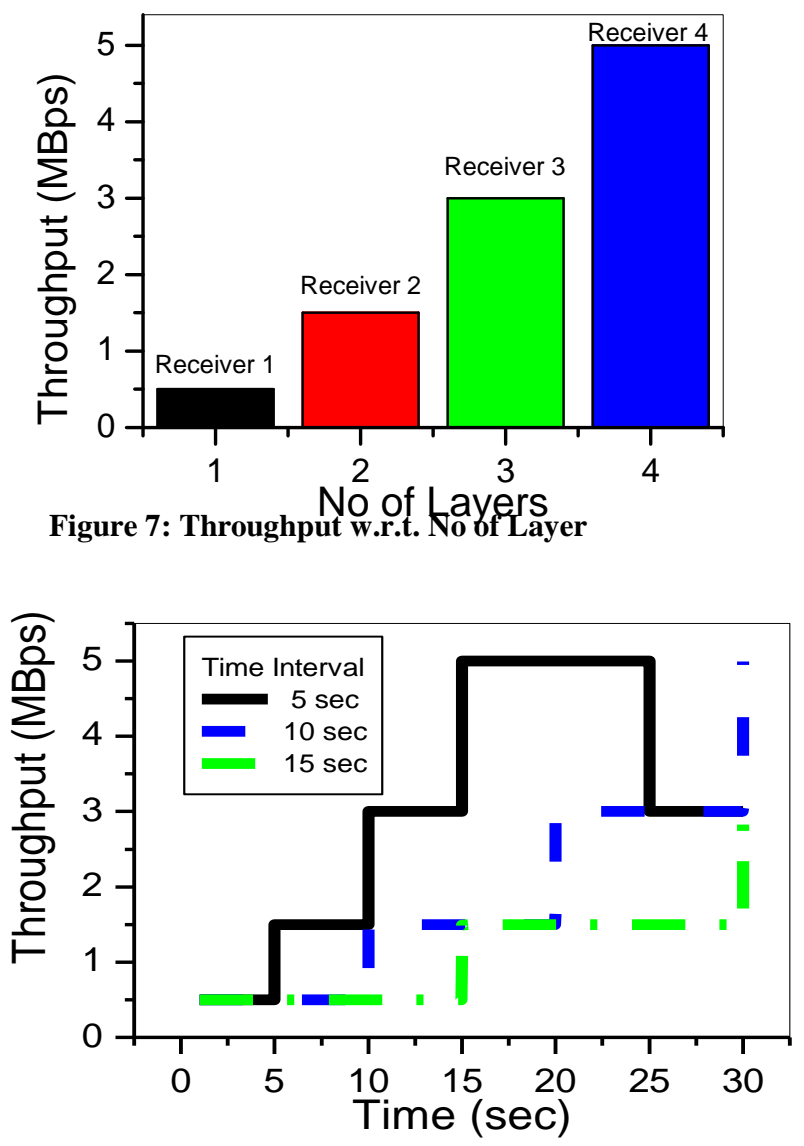

Figure 8: Throughput w.r.t. Time at $\mathbf{P}$ (T)

In figure 9 graphs plotted between throughput and time at at different value of $\rho$.

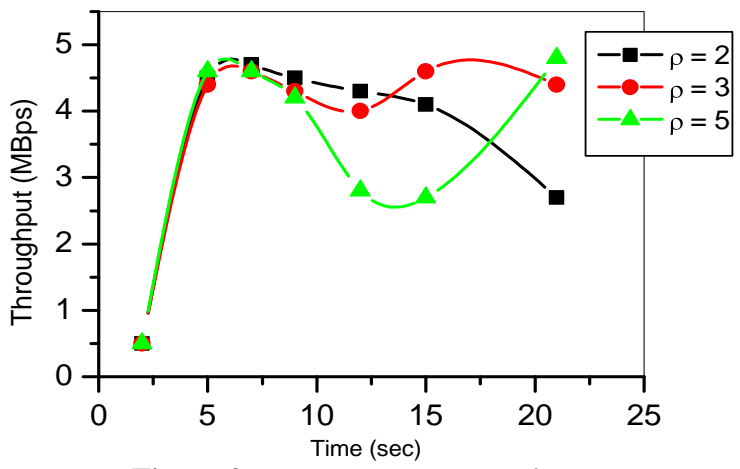

Figure 9: Throughput w.r.t. Time at $\rho$

The graph shows that at $\mathrm{p}=2$ receivers go in deaf stage for more time because overloaded factor value $\left(\mathrm{Th}_{\mathrm{Over}}\right)$ will be more. So packet loss are increasing slowly (not avoiding) which decreasing the throughput. At $p=3$ receiver may go in both phase (deaf and 
leaving) because overloaded factor is less. So, first throughput decrease slowly after some time throughput increases smoothly. At $p=5$ because overload factor are very less so it will reach in leaving or joining phase so throughput first decrease and after some time increase sharply.

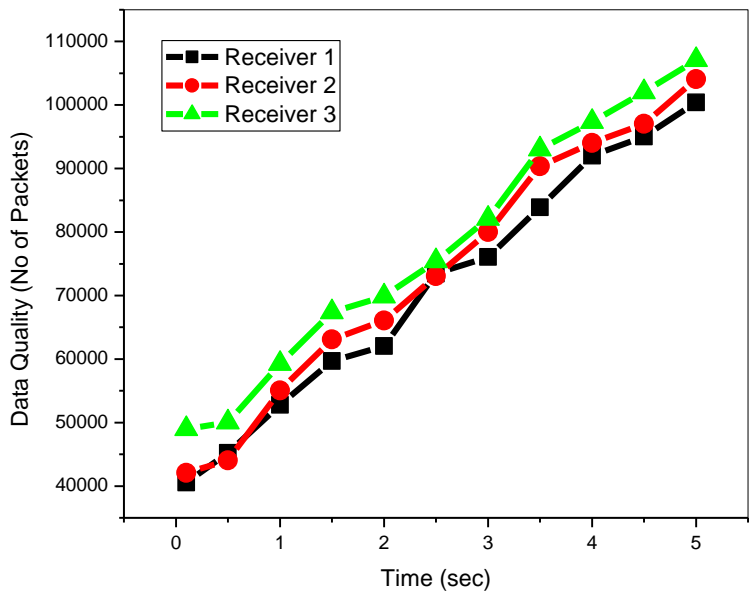

Figure 10: Data Quality w.r.t. Time

In figure 10 graphs plotted between data quality (receive number of packets) and time which shows current status of data quality received by different receivers. At time $1.5 \mathrm{~s}$ when receiver are $1,2,3$ which are receiving data from different layers than data quality are 58849, 63312 and 68129 packets respectively. The graph shows that if receivers receive more layers then data quality also increase receptivity.

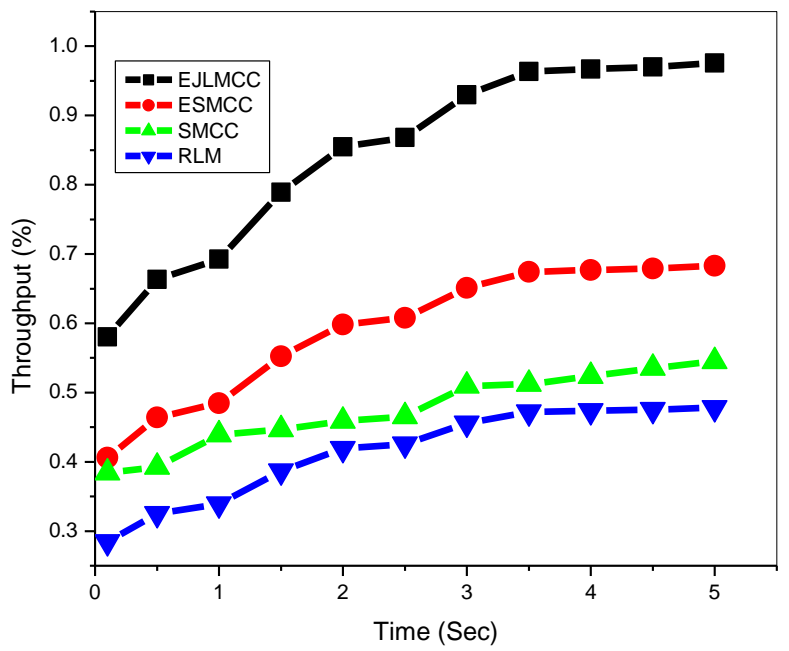

Figure 11: Throughput w.r.t. Time

In figure 11 graphs plotted between throughput and time which shows improvement in throughput of proposed approach with respect to existing schemes. We can observe from graph that proposed approach (EJLMCC) is better than existing one. Because we are take decision according to adaptive throughput which helps to increase the throughput due to joining multilayer instead of single layer while it recover the packet loss using deaf concept and maintain the throughput. Here at time $3 \mathrm{~s}$, our approach throughput is $47 \%$ more that RLM while $36 \%$ and $27 \%$ more than SMCC and ESMCC respective.

\section{CONCLUSION}

We proposed a new adaptive approach based on available throughput using efficient join and leave operation. In proposed approach the receiver join the multiple layers instead of single layer at a time and use the adaptive leaving decision for deaf or leave layer. Multiple layer joining increase the throughput of receiver while deaf concepts to avoid the layer leaving which reduce the packet loss. The simulations result indicates that our proposed approach is better than existing approach. We achieved improvement in term of throughput which increases the data quality of applications.

\section{APENDIX A}

In this appendix, we are providing the complete algorithm for joining and leaving operation.

\section{Proposed Algorithm}

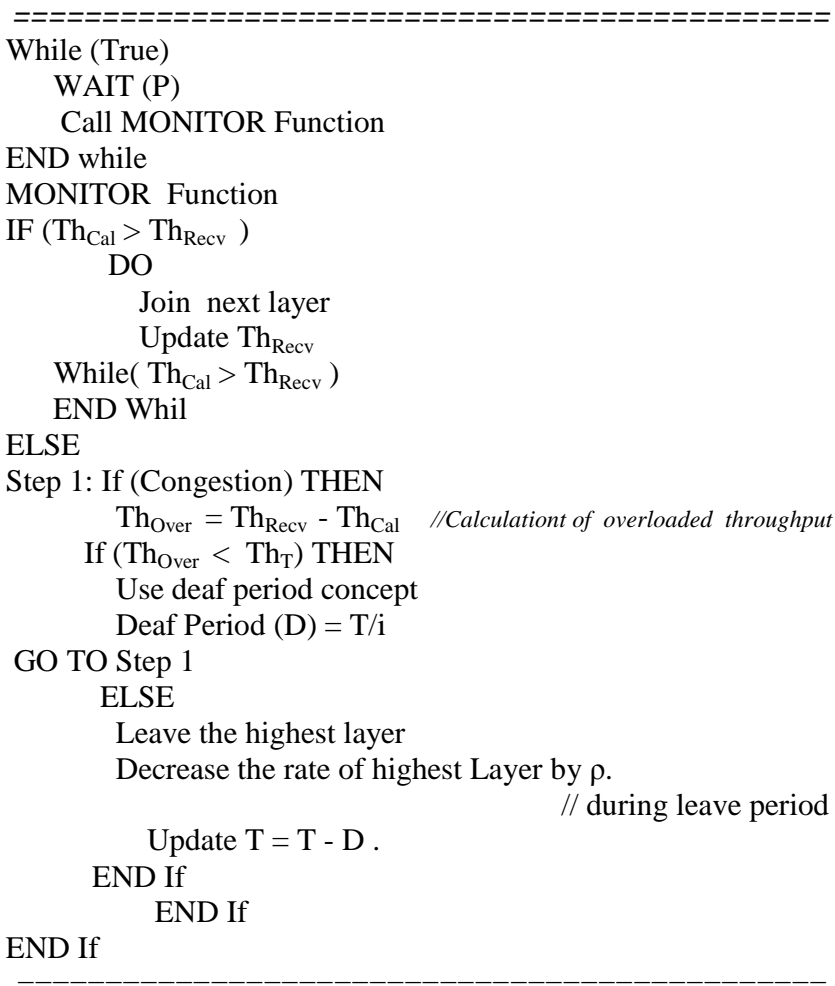

\section{REFERENCES}

[1] C. Yang and A. V. S. Reddy.: A Taxonomy for Congestion Control Algorithms in Packet Switching NetworksIEEE Network, vol 5, pg.34-4, 1995.

[2] Congestion Control in Computer Networks: Issues and Trends, IEEE Network Magazine, pg.24-30, May 1990.

[3] Cote, G., Erol, B., Gallant, M. and Kossentini, F., H.263+.: Video Coding at Low Bit Rates, IEEE Transactions on Circuits and Systems for Video Technology, 8(7), Nov. 1998, 849-866.

[4] D.S. Yin, Y.H. Liu, et al..: "A new TCPfriendly congestion control protocol for layered multicast", in proc. IASTED 
conference on Internet and Multimedia Systems and Applications, Innsbruck, Austria, Feb. 2006.

[5] G. Kwon and J. Byers, "Smooth multirate multicast congestion control," Technical Report BU-CS-TR-2002-025, Boston Univ. 2002.

[6] J. Byers, M. Frumin, et al., "FLID-DL: congestion control for layered multicast", in Proc. NGC2000, Palo Alto, USA, pp.71-81, Nov. 2000

[7] Karan Singh with Rama Shankar Yadav"A SURVEY ON MULTICAST CONGESTION CONTROL" Proceedings of Third Innovative Conference on Embedded Systems, Mobile Communication and Computing $\left(\right.$ ICEMC $^{2}$ ), pp 186-197, August, 2008

[8] Kulatunga, Fairhurst "TFMCC Protocol Behaviour in Satellite Multicast with Variable Return Path Delays" IEEE 2006.

[9] Legout, A., Biersack, E. W.: PLM: Fast Convergence for Cumulative Layered Multicastb Layered Multicast ransmission. Proceedings of ACM SIGMETRICS, June 2000

[10] McCanne S., Jacobson V., and Vetterli M.: Receiver-driven layered multicast, Proceedings of ACM SIGCOMM, pp.117130, August 1996, New York, USA.

[11] ns2: http://www.isi.edu/nsnam/ns/

[12] Qian Zhang, Quji Guo, Qiang Ni, Wenwu Zhu, and Ya-Qin Zhang, Source adaptive multi-layered multicast algorithms for real-time video distribution, IEEE/ACM Transactions on Networking, vol.8, no.6. pp.720-733, 2006.

[13] Sisalem, Wolisz "MLDA: A TCP-friendly Congestion Control Framework for Heterogeneous Multicast Environments" IEEE 2000.
[14] Stian Johansen, Anna N. Kim, Andrew Perkis.:"Quality Incentive Assisted Congestion Control for Receiver-Driven Multicast” IEEE Communications Society ICC 2007.

[15] W. Kammoun, H. Youssef "An adaptive Mechanism for End-to-End Multirate Multicast Congestion Control"in proceeding of The Third International Conference on Digital Telecommunications pp 88-93, 2008.

[16] Li, B., Liu, J.: Multirate video Multicast over the Internet: An Overview. IEEE Network. January/February 2003.

[17] B. Fenner et. Al. "Internet Group Management Protocol (IGMP)" Request for Comments 4605, August 2006.

[18] Deering. S.: Multicasting Routing in Internetwork and Extended LANs, SIGCOMM Summer 1988 Proceeding, Aug 1988.

[19] Distance Vector Multicast Routing Protocol, Request for Comments 1075, November 1988.

[20] S. E. Deering et. Al.: "Host extensions for IP multicasting", Request for Comments 988, July, 1986.

[21] J. Widmer and M. Handley, "Extending equation basedcongestion control to multicast applications," in Proc. of ACM SIGCOMM '01.

[22] L. Rizzo. "A TCP-friendly single-rate multicast congestion control scheme", in Proc. ACM SIGCOMM, pp.17 28,Stockholm, Sweden, August 2000.

[23] S. Floyd, M. Handley, J. Padhye, and J. Widmer "Equationbased congestion control for unicast applications" in Proc. ACM SIGCOMM, pages 43 - 56, Stockholm, Sweden, Aug. 2000. 